\title{
MODIFIED TRAJECTORY TRACKING GUIDANCE FOR ARTILLERY ROCKET
}

\author{
Rafae Ożóg, Mariusz Jacewicz, Robert GŁębocki \\ Warsaw University of Technology, Warsaw, Poland \\ e-mail:rozog@meil.pw.edu.pl; mjacewicz@meil.pw.edu.pl; rglebocki@meil.pw.edu.pl
}

\begin{abstract}
This paper contributes to a modified guidance scheme based on a trajectory tracking method which is dedicated for an artillery rocket with a finite set of single use solid propellant side thrusters. Frequency modulation of pulses was used to achieve effective firing logic. The proposed control law is applicable in the last phase of flight when the rocket reaches the vertex of the trajectory. A correction engine activation sequence was chosen in such a way that possibility of rocket axial unbalance is minimized due to motors firing. Numerical simulation results indicate that significant dispersion reduction was achieved and the number of activated side rocket thrusters is minimized.
\end{abstract}

Keywords: trajectory tracking, guidance, lateral thruster

\section{Introduction}

Rocket artillery systems have been commonly used for years on the battlefields. The main roles of this kind of weapon are: preparation of the area before the main ground troops attack, fire support for other types of forces and defensive tasks. The main advantages of these systems are low unit cost and strong firepower. Among the disadvantages of unguided rockets, one may distinguish their huge dispersion at long ranges and poor impact point accuracy, especially when launched at low elevation angles. Generally, rocket leaves the launcher with a relative low velocity, therefore, if some disturbances occur (e.g. wind shear) at the very beginning of the active portion of the trajectory then the final impact point might be positioned far away from the desired one. Due to this reason, rocket artillery is commonly used rather in a role of area weapon than a precision one. One of the current tendencies in modern warfare development and the significant requirement is to improve the rocket range. On the other hand, at long ranges the accuracy could be lost. Moreover, precision hit functionality is also needed especially at asymmetric conflicts to eliminate point targets and reduce losses among civilians.

One of the ways to improve the weapon effectiveness is to use cluster warheads. From another point of view, in a lot of countries this kind of munition cannot be used due to law restrictions like Convention of Cluster Munitions. One of the possibilities to achieve significant dispersion reduction is to acquire guided capabilities. Microelectromechanical Systems (MEMS) could be used to include a low-cost control mechanism into a rocket structure and then the unguided rocket can be turned into a high-performance precision weapon. Various actuators like movable fins, lateral thrusters, dual spin projectiles with forward canards and internal movable mass elements have been implemented up to this time. With the precision guidance, the unit cost of enemy neutralization could be decreased because the total number of rockets used per one target is smaller.

In this paper, a control system based on single use lateral thrusters is proposed in order to reduce rocket dispersion. There exist a lot of problems while this type of control is utilized. In the case of aerodynamic fins, the task is much easier due to the fact that the rocket trajectory 
could be changed in a continuous manner. When the pulse jet mechanism is used, there is no possibility to control rocket motion continuously. There exists only a finite number of control pulses which can be generated by lateral thrusters to influence on rocket trajectory. It was decided that to overcome these tasks, a new guidance scheme should be investigated.

\section{State of the art}

Different kinds of control methods have been published so far. The simplified trajectory tracking scheme was proposed in (Jitpraphai and Costello, 2001). The influence of specific parameters of lateral thrusters and the control system like total impulse and tracking window size on the accuracy were investigated. A significant dispersion reduction was achieved with this kind of method but no analysis about time between two pulses was presented. Next, the trajectory tracking guidance scheme with proportional navigation and with parabolic proportional guidance was compared in (Jitpraphai et al., 2001). The authors of that work concluded that proportional navigation allows achieving the least dispersion reduction when compared to two other methods. The trajectory tracking method generated low dispersion and was easily implementable on an onboard computer. In (Pavkovic et al., 2012), the method of calculating the time between two consecutive pulses was described. A simple active damping method, which allows counteracting the effects of disturbances at the beginning of the active portion of the trajectory was proposed in (Pavkovic, 2012). A flight path steering method, which was based on a pitch autopilot and control fins, was described in (Mandić, 2009). In (Cao et al., 2013) the authors proposed to use a lateral thrusters correction kit and laser seeker for a $120 \mathrm{~mm}$ projectile. They concluded that the trajectory errors were reduced by impulse thrusters. A roll autopilot design methodology for a canard controlled $122 \mathrm{~mm}$ artillery rocket using state-dependent Riccati equation method was presented in (Siddiq et al., 2012). A set of control laws based on proportional navigation with a pulse jet control mechanism was investigated in (Pavic et al., 2015). The authors achieved a drastic reduction of the mortar munition dispersion. An influence of basic control system parameters in the trajectory tracking guidance scheme was discussed in (Gupta et al., 2008). An optimum control scheme for a thruster-based correction kit was considered in (Gao et al., 2015).

It is possible to utilize some standard methods which are suitable for this kind of problem. The first group of control algorithms is based on the reference trajectory tracking. In this method, the rocket is moving along a prespecified curve. The second group of algorithms is based on the impact point predictors. In this approach, the instantaneous impact point to the ground is predicted during the flight, and the trajectory corrections are made. Sometimes these two types of control are mixed to achieve the best accuracy. Both methods could be also used at different flight phases to achieve the best possible performance (for example M270 Multiple Launch Rocket System uses the trajectory tracking scheme until the apogee and impact point prediction at the descent portion of the trajectory).

\section{Modified trajectory tracking guidance}

In this Section, the developed method is described. At the beginning, the main factors influencing the rocket accuracy are considered. Next, a test platform is described shortly. Finally, a control law is proposed.

\subsection{Dispersion factors}

One of the most current requirements for rocket artillery is to achieve the maximum range with a minimum dispersion. These two issues are contradictory. The longer the rocket range, 
the lower accuracy it achieves. The dispersion is influenced and generated by a huge set of disturbances, which acts on the rocket during the whole flight. The most significant factors at the launch phase are: initial elevation and azimuth launch angles of the launch tubes, launcher vibration while the rocket moves along a tube, the firing order from launch tubes, tubes location axial misalignments and the time between two consecutive firings. Furthermore, the total impulse variation of the rocket motor is the main factor which influences the longitudinal dispersion and achieved range. This factor could be reduced only at the manufacturing stage. Thrust misalignment effects are partially mitigated due to rocket spinning around their longitudinal axis. A lot of artillery rockets are stabilized with the aim of deployable fins. Fin angles uncertainties could influence the maximum spin rate along longitudinal axis and decide about lateral dispersion. Surface properties of the rocket fuselage (surface roughness) could be taken into account. Finally, the wind during the flight determines the achieved dispersion.

\subsection{Test platform and simulation model}

The geometric and mass data of one of the existing artillery rocket were used as an input for numerical simulations. The rocket diameter was $128 \mathrm{~mm}$ and the length was equal to $2.6 \mathrm{~m}$. The rocket mass, center of mass location measured from tail, axial and transversal moments of inertia were assumed to be approximately $60.75 / 38.55 \mathrm{~kg}, 1.44 / 1.65 \mathrm{~m}, 0.14 / 0.09 \mathrm{kgm}^{2}, 38 / 29 \mathrm{kgm}^{2}$ before and after the burnout, respectively. The rocket has four wrap-around fins at the tail. The maximum range was more than $47 \mathrm{~km}$ when launched on high elevation angles. It was proposed to use the trajectory correction only when the axial spin frequency was smaller than $12 \mathrm{~Hz}$.

For the purposes of numerical experiments, a 6 degrees of freedom (6-DOF) mathematical model has been used to investigate the rocket behavior (McCoy, 2004; Zipfel, 2014; Żugaj and Głębocki, 2010). The nondimensional forces and moments aerodynamic coefficients were obtained with the aim of semi-empirical engineering methods (Moore, 1993). Next, the aero database was validated using FLUENT CFD code. A lookup-table procedure was used to construct the aero database. The main rocket motor characteristics were obtained for three different temperatures $\left(-40^{\circ} \mathrm{C},+20^{\circ} \mathrm{C}\right.$ and $\left.+50^{\circ} \mathrm{C}\right)$ on a test stand. The base drag connected with the main engine state (on or off) was also included in the model. The time of the main rocket motor burnout was approximately $3.7 \mathrm{~s}$. The total impulse of the engine was assumed to be $60000 \mathrm{Ns}$.

It was assumed that the information about rocket position and angular rates was available from a Strapdown Inertial Navigation System (3 accelerometers, 3 gyroscopes and magnetometer) integrated with a sun sensor (photodiode). No disturbances from sensors were considered in numerical simulations.

The control system is composed of 30 solid propellant small rocket motors (Fig. 1). The lateral thrusters system consists of a ring of small thrusters mounted in the nose section. The motors are equally spaced around the fuselage. The guidance kit is mounted approximately $2 \mathrm{~m}$ from the rocket tail, before the center of gravity of the rocket. The angle between two engines is $12^{\circ}$. Every motor can be used only once, what is the basic constraint on the control algorithm. The total impulse of the lateral thruster and time of its work are also fixed. The most important parameter which can be modified after launch is the time between two consecutive pulses. The influence of igniter is also included in the model. The operation time of the single thruster is $0.02 \mathrm{~s}$ with standard deviation equal to $0.003 \mathrm{~s}$ while the maximum available thrust is $900 \mathrm{~N}$.

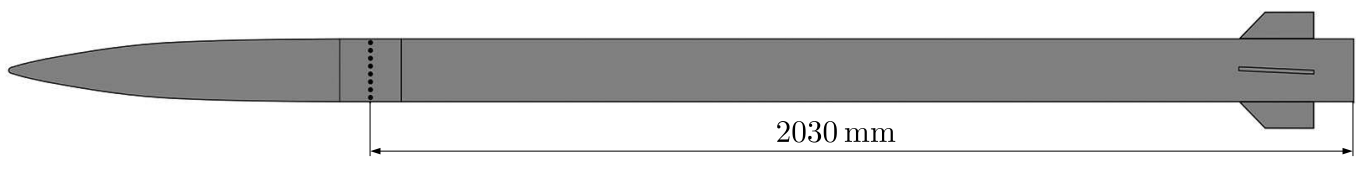

Fig. 1. Lateral thrusters location 
The vast majority of flight control solutions can generate lateral control forces in a continuous way. The analyzed solution fall into category recognized as the pulse control systems.

\subsection{Control system}

The control system is composed of two subsystems: the control law subsystem and firing-logic subsystem. The main task of the control system is to decide whether or not the trajectory should be changed. This subsystem generates the initial pulses one by one. The firing-logic circuit decides which pulse jet should be used firstly. The main task of this system is to decide which thruster should be fired.

In "state of the art" Section, only one author from cited works taking in advance the thrusters firing sequence. Most of the authors ignore this important issue and assumed that the neighboring motors are fired one by one in a sequence. When a lot of engines are fired only from one side of the rocket, the object might fall into axial unbalance which can lead to rocket destruction due to intensive vibrations. To prevent this scenario, a firing logic was developed (Fig. 2). It is assumed that at the very beginning, engine number 1 is fired. In the next, step engine 2 at the opposite side of the fuselage is activated and the whole sequence is repeated until all engines are consumed.

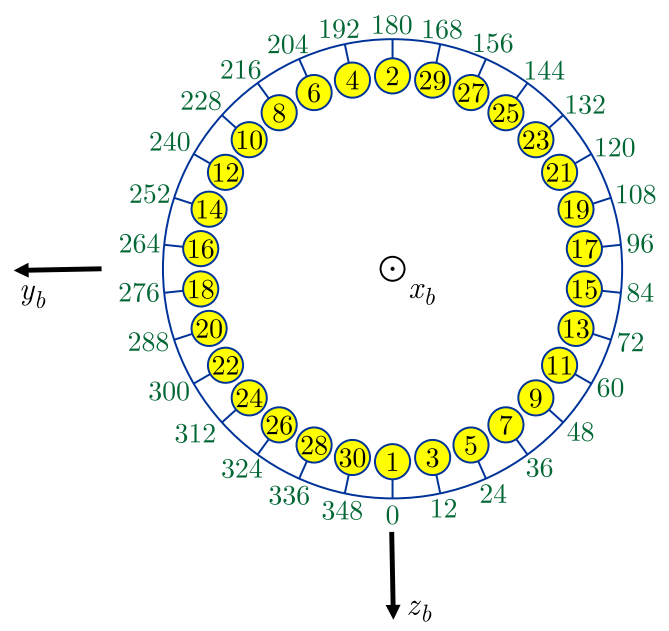

Fig. 2. Lateral thruster firing order sequence

It is assumed that the thrust force of each lateral thruster is defined in the manufacturing stage and cannot be modified during the flight. Furthermore, the time of the work of the single thruster is constant. Therefore, the only possibility to influence rocket motion is to modify the time $\tau$ between two consecutive pulses. The proposed algorithm is based on the trajectory tracking method. One of the most significant issue is to perform a proper reference trajectory generation scheme. In this work, a simplified method was used and it was assumed that the reference trajectory is generated for a rocket without any disturbances (nominal main motor specific impulse, no thrust misalignment, etc.). This set of reference trajectory data can be implemented into the onboard computer before the rocket launch.

The error between the reference trajectory and actual position on the flight path has been estimated as a difference between coordinates of a point for a given time $t$ which has passed from the rocket launch. Next, the error is transformed to the body coordinate system (Jitpraphai and Costello, 2001)

$$
\left[\begin{array}{l}
e_{n x}(t) \\
e_{n y}(t) \\
e_{n z}(t)
\end{array}\right]=T_{n}^{b}\left[\begin{array}{l}
x_{\text {nref }}(t)-x_{n}(t) \\
y_{\text {nref }}(t)-y_{n}(t) \\
z_{\text {nref }}(t)-z_{n}(t)
\end{array}\right]
$$


where

$$
T_{n}^{b}=\left[\begin{array}{ccc}
\cos \Theta \cos \Psi & \cos \Theta \sin \Psi & -\sin \Theta \\
\sin \Phi \sin \Theta \cos \Psi-\cos \Phi \sin \Psi & \sin \Phi \sin \Theta \cos \Psi+\cos \Phi \cos \Psi & \sin \Phi \cos \Theta \\
\cos \Phi \sin \Theta \cos \Psi+\sin \Phi \sin \Psi & \cos \Phi \sin \Theta \sin \Psi-\sin \Phi \cos \Psi & \cos \Phi \cos \Theta
\end{array}\right]
$$

and $\Phi, \Theta, \Psi$ are roll, pitch and yaw angles, respectively. The main disadvantage of this simplified method is that there exists a longitudinal error between the position on a reference path at the current time $t$ and the actual position of the rocket. In other words, the rocket could be in front of or behind the point in which should it be at the time $t$. It is possible to calculate directly the perpendicular distance to the desired rocket path but this procedure might be time consuming and leads to undesirable high requirements for the onboard computer, which increases the total guidance unit cost.

It is assumed that the error location of the rocket due to the reference trajectory will be expressed in polar coordinates in the plane perpendicular to the longitudinal axis of the rocket. Next, two pieces of information are needed to specify the rocket position base on the reference trajectory: amplitude and phase of the error location. The amplitude error $\Gamma$ is defined as

$$
\Gamma=\sqrt{e_{n y}^{2}+e_{n z}^{2}}
$$

The $\Gamma$ gives information how far from the reference trajectory the rocket is. The phase error $\gamma$ expresses the angular position of the rocket in relation to the reference path

$$
\gamma=\bmod \left(-\arctan 2 \frac{e_{n y}}{e_{n z}}, 2 \pi\right)
$$

In a general case, the thruster system can consists of $M$ rings of motors with $N$ thrusters in each ring. The matrix $\mathbf{S}$ is introduced to describe which from the lateral thrusters have already been fired. The matrix is defined as

$$
\mathbf{S}=\left[\begin{array}{ccc}
S_{1-1} & \cdots & S_{1-M} \\
\vdots & \ddots & \vdots \\
S_{N-1} & \cdots & S_{N-M}
\end{array}\right]
$$

The number of rows in this matrix is equal to the number of lateral thrusters in each ring, and the number of columns is equal to the number of motor rings. Each element $S_{i-j}$ corresponds to the $i$-th engine in the $j$-th layer and could take two values: 0 - engine not already fired or 1 - engine already fired. In the analyzed case, the dimension of this matrix is $30 \times 1$. After engine burnout, the $S_{i-j}$ value is changed instantaneously from 0 to 1 .

Next, a set of conditions in which the lateral thruster should be activated are introduced. The conditions proposed by some authors, who were cited at the beginning of this article, are similar. Here, additional conditions when the control system should be activated are introduced. They are as follows:

- The lateral thruster $i-j$ has not been already fired, so the $S_{i-j}$ is equal to zero.

- The error $\Gamma$ is bigger that a certain specified threshold value.

$$
\Gamma \geqslant r_{\text {thres }}
$$

where $r_{\text {thres }}$ is the tracking window size. The above mentioned condition means that when the rocket is inside the corridor of radius $r_{\text {thres }}$, no control action is performed. A set of some small values (order of several meters) of this parameter is investigated to choose the best accuracy. For $r_{\text {thres }} \rightarrow 0$ control action is required even if the real distance error $\Gamma$ is very small, which can lead to undesirable thruster consumption. On the other hand, for $r_{\text {thres }} \rightarrow \infty$, the control system is practically inactive during the whole trajectory. 
- The time from the previous rocket engine firing is longer than $\tau$

$$
t-t_{\text {prev }} \geqslant \tau
$$

where $t$ is the actual flight time, $t_{\text {prev }}$ is the last moment in which one of the thrusters has been fired and $\tau$ is the threshold parameter which could be tuned during the flight to achieve the best possible control quality.

- The thruster which should be fired as next is located exactly in the opposite direction of the fuselage than the desired lateral rocket movement (Fig. 3)

$$
\left|\gamma-\Phi_{i-j}-\pi-P\left(\tau_{d}+\tau_{s k}\right)\right| \leqslant \varepsilon
$$

where $\Phi_{i-j}$ is the angle of the $i-j$-th engine, $\gamma$ is the error defined by equation, $P$ is the rocket roll rate, $\tau_{d}$ is lateral thruster igniter delay and $\tau_{s k}$ is a half of pulse duration. The $\varepsilon$ is the activation threshold, and its values should be of an order of a fraction of a degree to several degrees. The $\Phi_{i-j}$ angle is changed after each rocket firing. The term $P\left(\tau_{d}+\tau_{s k}\right)$ describes the delay of the thruster initiator.

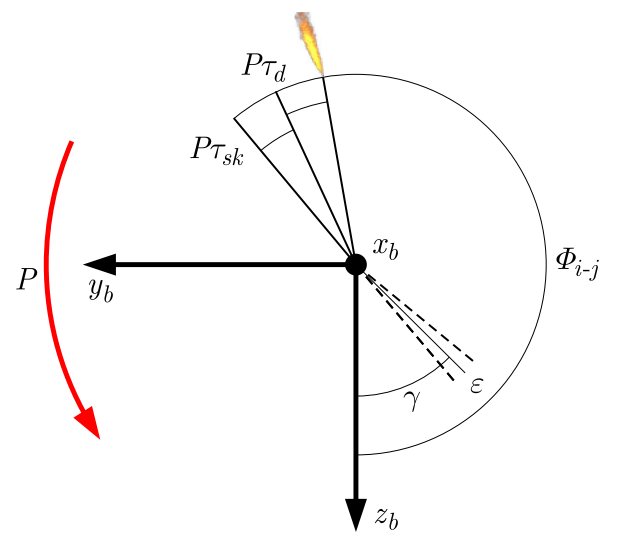

Fig. 3. Definition of roll angles (view from the rocket nose)

- The global condition in which the flight phase control system should be active could be mathematically expressed as a time after which the system is activated. The other possibility is to constraint the work of the system with the roll rate. The lateral thruster cannot be fired when the rolling rate is too high due to its effectiveness. The maximum roll rate of the test platform was approximately $25 \mathrm{rev} / \mathrm{s}$. It was decided that the thruster would be fired only when the angular rate would be smaller than $10 \mathrm{rev} / \mathrm{s}$. The other limit could be formulated with the aim of the rocket pitch angle $\Theta$ for which the control system was activated. Finally, it was decided that the lateral thrusters would be activated only when the rocket pitch angle $\Theta$ was smaller or equal to a prespecified threshold $\Theta_{g}$ value, i.e.

$$
\Theta \leqslant \Theta_{g}
$$

It means, that up to this time, there will be no control action. The control law based only on the above mentioned conditions has tendency to track precisely the predefined trajectory rather than to steer the rocket in the direction of the target which can lead to unnecessary thruster consumption. This is illustrated in Fig. 4. The passive portion of flight starts at the point $A$. Up to the point $B$ no control is used, because the pitch angle $\Theta$ is bigger than the threshold value $\Theta_{g}$. Starting from the point $B$, when all activation conditions are satisfied, the lateral thrusters are fired and the rocket is steered to the reference trajectory, so the tracking 
error decreases to the point $C$. When the control is continued in this way, the error $\Gamma$ still decreases and hit the horizontal line in the point $F$. Then, the error might increase again, and there is possibility that the target $\mathrm{E}$ will be not eliminated. To prevent this effect, the nature of the control algorithm operation should be changed immediately when the point $C$ is reached. Between the points $C$ and $E$ it is much more important to steer the rocket in such a way that the tracking error curve should hit the point $E$ at the end of the flight. So, the error should be eliminated just before target hitting rather than in the whole time range.

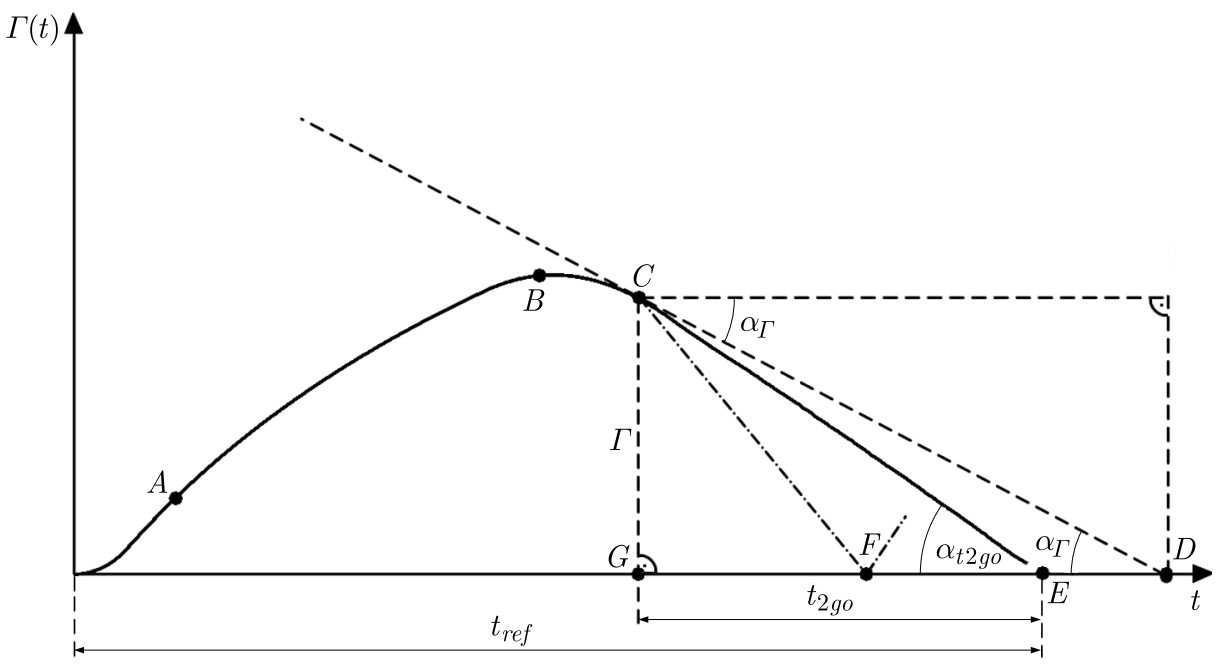

Fig. 4. Trajectory tracking error

In order to realize this concept, an additional condition was introduced. At first, the time-to-go $t_{2 g o}$ was calculated as a difference between the total time of flight $t_{r e f}$ and the time $t$ which lasted from the beginning of flight

$$
t_{2 g o}=t_{\text {ref }}-t
$$

Next, using the trigonometric function, the slope of the error curve $\Gamma$ in the point $C$ was defined in the form

$$
\alpha_{\Gamma}=-\arctan \frac{d \Gamma}{d t}
$$

The minus sign in the above formula results from the fact that the positive value of $\alpha_{\Gamma}$ was defined for the negative line slope, when the error amplitude decreased. In this way, (3.3) describes the error amplitude, and (3.11) takes into account steepness of the error curve $\Gamma$. From another point of view, the angle $\alpha_{t 2 g o}$ between the time axis and line segment $\mathrm{CE}$ was calculated as

$$
\alpha_{t 2 g o}=\arctan \frac{\Gamma}{t_{2 g o}}
$$

The maximum value of this angle might be $90^{\circ}$. Lateral thrusters should be used only when the absolute value from difference between (3.12) and (3.11) is greater than or equal to a certain threshold

$$
\left|\alpha_{t 2 g o}-\alpha_{\Gamma}\right| \geqslant \alpha_{\Gamma \text { thres }}
$$

where $\alpha_{\Gamma \text { thres }}$ is some small value, for example $0.1^{\circ}$. If $\alpha_{\Gamma} \leqslant \alpha_{t 2 g o}$, then the rocket is steered to the reference trajectory. Similarly, when $\alpha_{\Gamma}>\alpha_{t 2 g o}$, then the control system tries to move the center of mass of the rocket away from the reference path to prevent the situation as in the point $F$.

The above set of conditions were implemented into numerical simulation and tested to obtain its performance. 


\section{Simulation results}

In this Section, the results of numerical experiments were described. The 6-DOF mathematical model of the rocket was implemented into a MATLAB/SIMULINK software. The fixed-step ODE3 (Bogacki-Shampine) solver was used to integrate the equation of motion of the rocket numerically with the step size $0.0001 \mathrm{~s}$. Simulations were carried out on a standard desktop PC (Intel i7, 16 GB RAM).

Three kinds of simulations were considered. The first type was the nominal command trajectory which could be implemented in the onboard computer, and this trajectory should be followed by the guided rocket. The second type was an unguided flight path. In this experiment, the initial error in launching conditions was introduced to model the effect of rocket dispersion. No lateral motors were fired in this case. The initial launch parameters (Table 1) were assumed using data from the literature for similar objects (Gupta et al., 2008; Jitpraphai et al., 2001). The muzzle velocity was assumed to be decreased by $0.4 \mathrm{~m} / \mathrm{s}$ relative to the nominal case. The initial roll rate was also disturbed by $12^{\circ} / \mathrm{s}$. The initial elevation of the launch tube was set to $20^{\circ}$. This case was chosen to simulate the control effectiveness at low elevation angles, which is critical in the case of rocket artillery. The initial disturbances were $0.1^{\circ}$ in the launch tube elevation and $0.3^{\circ}$ in its azimuth. The total impulse of the rocket main motor was disturbed by $0.2 \%$ as well.

Table 1. Nominal and disturbed initial parameters used in simulations

\begin{tabular}{|l|c|c|c|}
\hline \multicolumn{1}{|c|}{ Parameter } & Nominal & Disturbed & Unit \\
\hline \hline Initial velocity & 32 & 31.6 & $\mathrm{~m} / \mathrm{s}$ \\
\hline Roll rate & 812 & 800 & ${ }^{\circ}$ \\
\hline Pitch rate & 0 & 0.01 & $\circ / \mathrm{s}$ \\
\hline Yaw rate & 0 & 0.007 & ${ }^{\circ} / \mathrm{s}$ \\
\hline Launch tube elevation & 20 & 20.1 & ${ }^{\circ}$ \\
\hline Launch tube azimuth & 0 & 0.3 & ${ }^{\circ}$ \\
\hline Total impulse & 100 & 99.8 & $\%$ \\
\hline
\end{tabular}

In the third case, the controlled path was the rocket trajectory when the lateral thrusters were fired to align the actual path to the desired. Due to rocket dynamical properties (relatively low stability margin), the control system was activated only in the descending portion of the rocket trajectory. The side motor thrust force was modeled as a rectangular pulse with time duration $0.02 \mathrm{~s}$ and amplitude $900 \mathrm{~N}$. The trajectory tracking window size $r_{\text {thres }}$ was $1 \mathrm{~m}$ and $\alpha_{\Gamma}=2^{\circ}$. The controlled trajectories were generated for various angles $\Theta_{g}$.

Figures 5 and 6 show trajectories of the rocket center of gravity during its motion in horizontal and vertical planes, i.e.: reference trajectory (blue solid line - "Reference" in figure legend), disturbed unguided (red dashed line - "Disturb. unc.") and disturbed guided (other lines). The black star at the reference trajectory means the rocket apogee. The achieved range was approximately equal to $24 \mathrm{~km}$. Up to $15 \mathrm{~km}$, there was no control action. The control system was activated immediately after reaching the prespecified $\Theta_{g}$.

The unguided rocket deviation was $110 \mathrm{~m}$ from the desired hit point. The curvature of the trajectory was high in the first correction stage, but later the flight path projection on the horizontal plane was nearly linear. For $\Theta_{g}=-5^{\circ}$, the initial control action was very intensive and before the target hitting control system had to move away the rocket from the nominal path to reach the target. Using the control law the lateral impact error was reduced to the order of single meters. The proposed method was able to reduce the lateral error even if the correction started at the final stage of flight. 


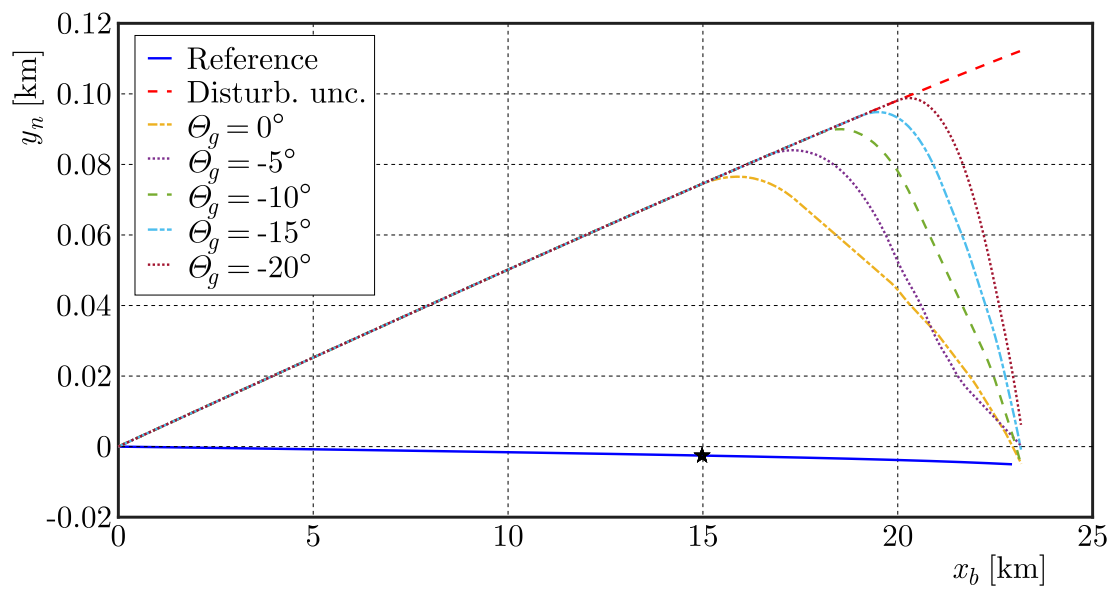

Fig. 5. Rocket trajectories in the horizontal plane

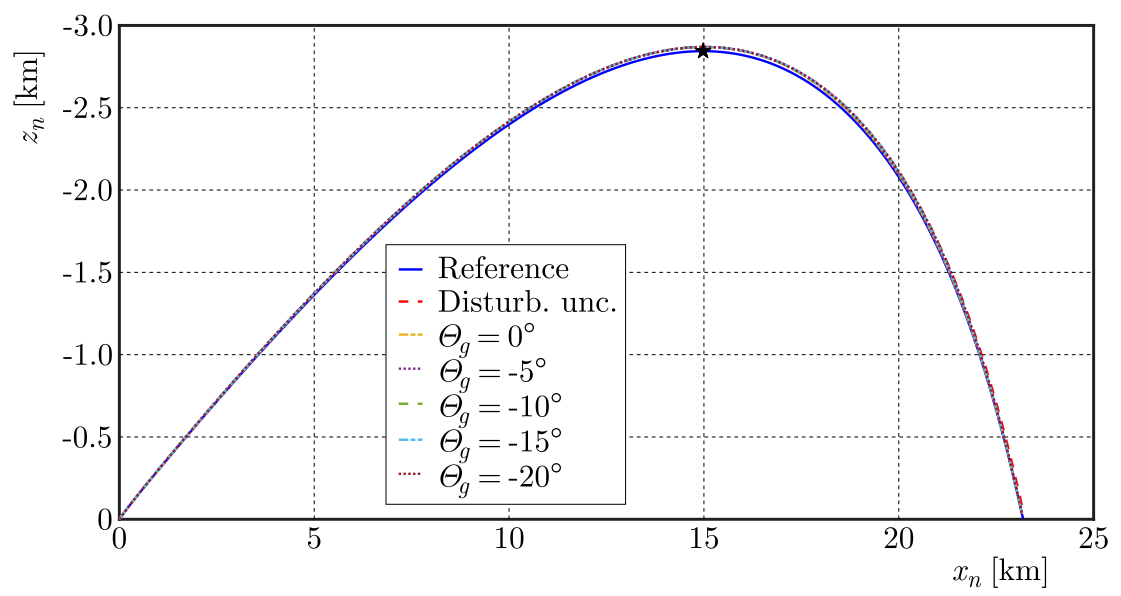

Fig. 6. Rocket trajectories in the vertical plane

The maximum height of flight was $2.8 \mathrm{~km}$ and occurred when the rocket traveled distance equal to $15 \mathrm{~km}$ in the horizontal plane. It may be observed that the disturbed trajectories were slightly above the nominal one. The disturbance in the initial pitch plane was chosen to be $0.1^{\circ}$, but even for such a small value all the corrected rockets hit the target with the accuracy of several meters.

In Fig. 7, the errors between the reference trajectory and the disturbed controlled and uncontrolled as a function of time were presented.

The longitudinal error was no bigger than $10 \mathrm{~m}$ and had opposite sign than in the disturbed uncontrolled case. Both errors, lateral and height, tended to zero when the control system was activated. When the lateral thrusters were activated too late, the last error was approximately $9 \mathrm{~m}$.

In Fig. 8, the distance errors, defined by equation (3.3), were compared. It is clearly visible that the control actions were performed after $15 \mathrm{~s}$, when the rocket reached the apogee point. By applying the proposed control law, the error was decreased smoothly, and at the impact time its value was smaller than $3.5 \mathrm{~m}$. In comparison with, for example, pure trajectory tracking guidance, which was mentioned in the "state of the art" Section, no oscillations were observed, which is desirable from the effectiveness point of view.

In Fig. 9, the number of thrusters firing as a function of time was presented. The total available number of thrusters was set to 30 . 


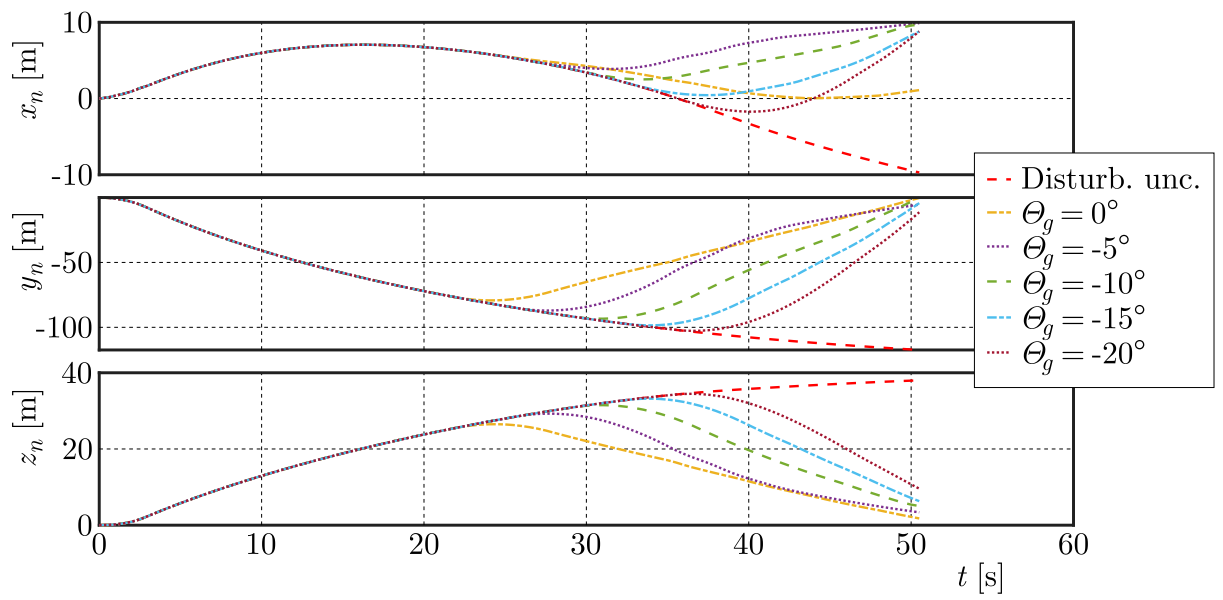

Fig. 7. Comparison of tracking errors

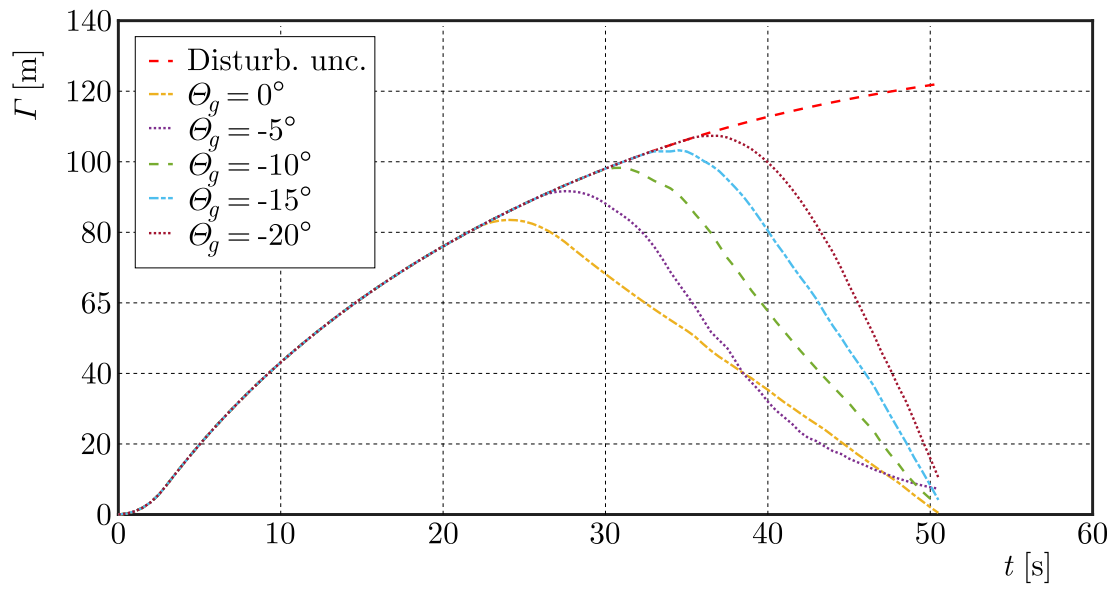

Fig. 8. Tracking error for various angles $\Theta_{g}$

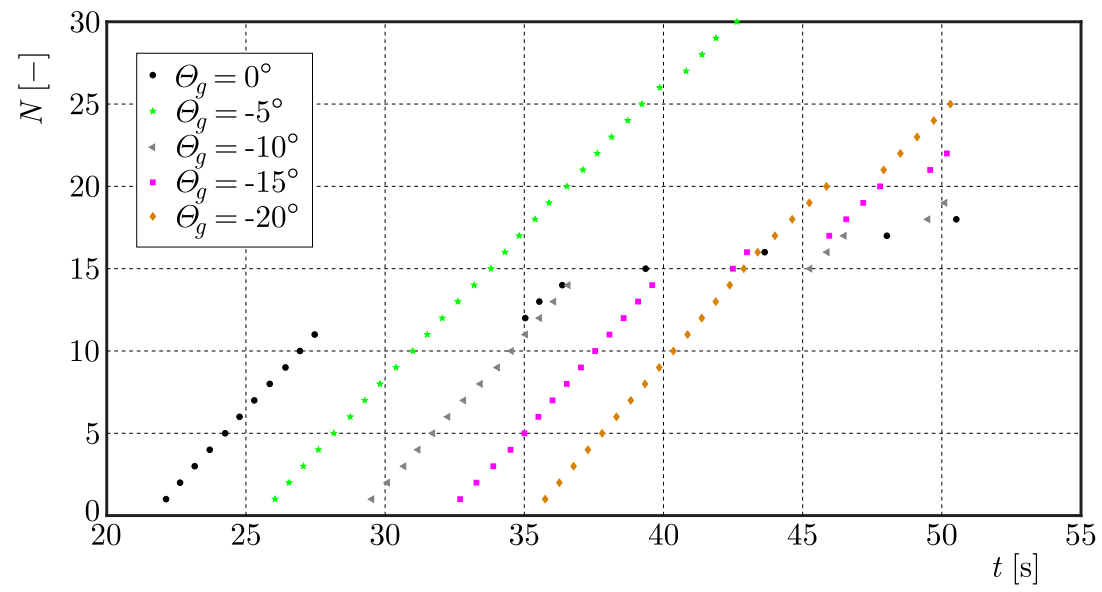

Fig. 9. Firing sequence of the pulsejet for a controlled trajectory

The control scheme worked in two operation modes. Before aiming the rocket to the target, the lateral thrusters were consumed as fast as possible with the prespecified $t_{\min }$. Next, the rocket was steered to the target with the use of single pulses. This effect was achieved while using the idea from Fig. 4. When $\Theta_{g}$ was equal to zero, 18 thrusters were consumed. The later the thrusters were activated, the bigger number of them was used. For $\Theta_{g}=-5^{\circ}$, all motors 
were consumed because at the beginning of guided phase the curvature of the tracking error was quite big due to too intensive control. It is worth nothing that the rocket was fired at a very low elevation angle, so at higher angles much more thrusters might be consumed due to longer flight time.

Finally, Monte-Carlo simulations were performed to determine the dispersion reduction capabilities of the proposed control scheme. 200 samples were used to generate dispersion patterns. Based on the data from (Pavic et al., 2015) and (Jitpraphai and Costello, 2001) the Gaussian distribution was used to introduce the uncertainty in each model parameter (Table 2). Marsenne-Twister algorithm was used to generate pseudorandom numbers. The CEP (Circular Error Probable) was used as a measure of dispersion. The CEP is a radius of a circle centered on the target for which the probability of failing of the projectile inside this circle is $50 \%$. A nonparametric median estimator of the CEP has been applied.

Table 2. Parameters used in Monte-Carlo simulations

\begin{tabular}{|c|c|c|c|c|}
\hline No. & Parameter & Mean value & Std. deviation & Unit \\
\hline \hline 1 & $m_{0}$ & 60.75 & 0.05 & $\mathrm{~kg}$ \\
\hline 2 & $m_{k}$ & 38.55 & 0.05 & $\mathrm{~kg}$ \\
\hline 3 & $I_{x 0}$ & 0.137 & 0.01 & $\mathrm{kgm}^{2}$ \\
\hline 4 & $I_{x k}$ & 0.091 & 0.01 & $\mathrm{kgm}^{2}$ \\
\hline 5 & $U$ & 32 & 0.9 & $\mathrm{~m} / \mathrm{s}$ \\
\hline 6 & $V$ & 1 & 0.5 & $\mathrm{~m} / \mathrm{s}$ \\
\hline 7 & $W$ & 1 & 0.5 & $\mathrm{~m} / \mathrm{s}$ \\
\hline 8 & $P$ & 1356 & 12 & $\circ / \mathrm{s}$ \\
\hline 9 & $Q$ & 0 & 1 & $\circ / \mathrm{s}$ \\
\hline 10 & $R$ & 0 & 1 & $\circ / \mathrm{s}$ \\
\hline 11 & $\Phi$ & 0.0 & 0.2 & $\circ$ \\
\hline 12 & $\Theta$ & 20 & 0.2 & $\circ$ \\
\hline 13 & $\Psi$ & 0 & 0.2 & $\circ$ \\
\hline
\end{tabular}

Figure 10a and 10b present a hitting pattern for the uncontrolled and controlled rocket, respectively.

(a)

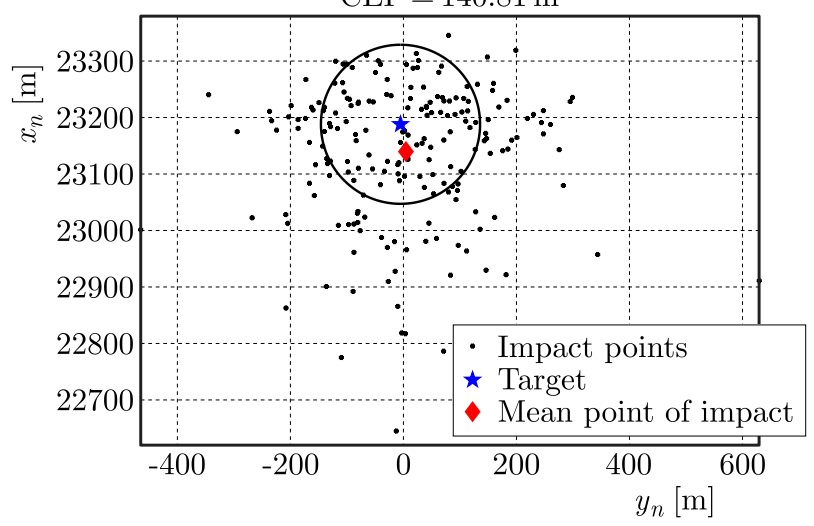

(b)

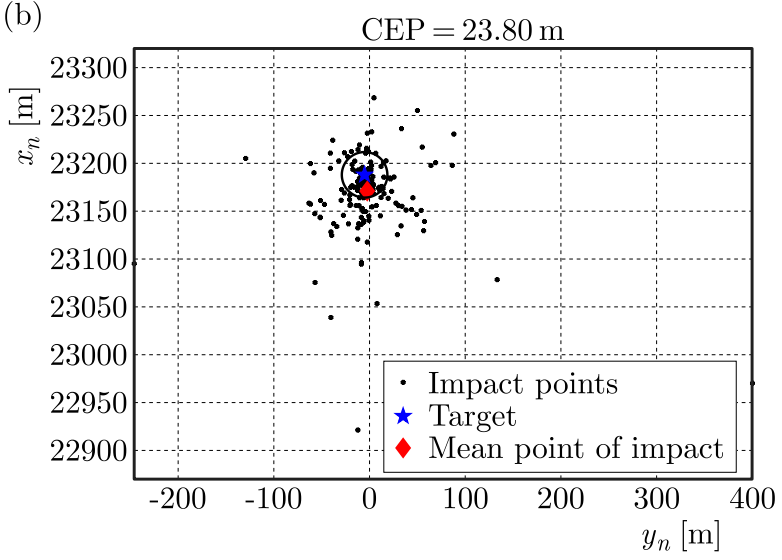

Fig. 10. Impact point dispersion of the rocket (a) unguided (b) with the control law for $\Theta_{g}=0^{\circ}$

The dispersion radius for the guided rockets was 5.9 times smaller than in the ballistic case. The Mean Point of Impact nearly coincided with the target when the control law was applied. 


\section{Conclusion}

In this paper, the firing logic for the rocket artillery was described. With the aim of numerical simulations, it was proved that the proposed method allows one to achieve a significant reduction in the dispersion of rocket impact points. The thruster firing order should be taken into account to prevent the rocket from the axial unbalance. The numerical experiments were conducted for a low elevation angle. The developed algorithm is quite sensitive to parameter variations like the minimum allowable time between two consecutive thruster firings and tracking window size. Monte-Carlo simulations proved that the main advantage of the proposed control law is the ability to significantly reduce dispersion of the rocket artillery.

Acknowledgements

This work was supported by The National Centre for Research and Development (NCBiR) under project DOB-BIO8/10/01/2016 "Projectiles control system technology development".

\section{References}

1. Cao X.B., Xu Y.C., Rui C.Y., Shan Z.Y., 2013, Simulation of trajectory correction for an impulse control mortar projectile with a strapdown laser seeker, Applied Mechanics and Materials, 367, 377-381

2. GaO M., Zhang Y., YAnG S., 2015, Firing control optimization of impulse thrusters for trajectory correction projectiles, International Journal of Aerospace Engineering, 2015, 1-11

3. Gupta S.K., Saxena S., Singhal A., Ghosh A.K., 2008, Trajectory correction flight control system using pulsejet on an artillery rocket, Defence Science Journal, 58, 1

4. Jitpraphai T., Burchett B., Costello M., 2001, A comparison of different guidance schemes for a direct fire rocket with a pulse jet control mechanism, AIAA Atmospheric Flight Mechanics Conference and Exhibit, Guidance, Navigation, and Control and Co-located Conferences

5. Jitpraphai T., Costello M., 2001, Dispersion reduction of a direct-fire rocket using lateral pulse jets, Journal of Spacecraft and Rockets, 38, 6, 929-936

6. Mandić S., 2009, Guidance of ground to ground rockets using flight path steering method, Scientific Technical Review, LIX, 3-4, 3-11, UDK: 623.465.3

7. Mccoy R., 2004, Modern Exterior Ballistics: The Launch and Flight Dynamics of Symmetric Projectiles, Schiffer Publishing, Ltd.

8. Moore F., 1993, State-of-the-art Engineering Aeroprediction Methods with Emphasis on New Semiempirical Techniques for Predicting Nonlinear Aerodynamics on Complete Missile Configurations, Naval Surface Warfare Center, Dahlgren, Virginia

9. Pavic M., Pavkovic B., Mandic S., Zivkovic S., Cuk D., 2015, Pulse-frequency modulated guidance laws for a mortar missile with a pulse jet control mechanism, The Aeronautical Journal, 119, 1213, 389-405

10. PAVkovic B., 2012, Enhancing the precision of artillery rockets using pulsejet control systems with active damping, Scientific Technical Review, 62, 2, 10-19, UDK: 621.453/457.001.57:519.637

11. Pavkovic B., Pavic M., Cuk D., 2012, Frequency-modulated pulse-jet control of an artillery rocket, Journal of Spacecraft and Rockets, 49, 2, 286-294

12. Siddiq M.K., FAng J.C, Yu W.B., 2012, State dependent Riccati equation based roll autopilot for $122 \mathrm{~mm}$ artillery rocket, International Journal of Aerospace and Mechanical Engineering, 6, 12, 2814-2822

13. Zipfel P., 2014, Modeling and Simulation of Aerospace Vehicle Dynamics, American Institute of Aeronautics and Astronautics, Inc.

14. ŻugaJ M., GŁęBocki R., 2010, Model of gasodynamic control system for guided bomb, Journal of Theoretical and Applied Mechanics, 48, 1, 27-44 